\title{
Capacitor-based Isolation Amplifiers for Harsh Radiation Environments
}

\author{
Francisco J. Franco*, Yi Zong, and Juan A. de Agapito* \\ Departamento de Física Aplicada III, Facultad de Ciencias Físicas, Universidad Complutense de Madrid, Ciudad \\ Universitaria, 28040 Madrid (Spain)
}

\begin{abstract}
Commercial-off-the-shelf (COTS) capacitor-based isolation amplifiers were irradiated at the Portuguese Research Reactor (PRR) in order to determine its tolerance to the displacement damage and total ionising dose (TID). The set of experimental data shows that some of these devices are suitable for zones inside future nuclear facilities where the expected total radiation damage would be below $2.2 \cdot 10^{13} 1-\mathrm{MeV}$ neutron $/ \mathrm{cm}^{2}$ and $230 \mathrm{~Gy}(\mathrm{Si})$. However, some drawbacks must be taken into account by the electronic designers such as the increase of the output offset voltage and the slight modification of the transmission gain.

Keywords: COTS, Displacement Damage, Isolation amplifiers, Total Ionising Dose (TID). PACS: 29.90.+r, 28.52.Lf, 28.41.Rc
\end{abstract}




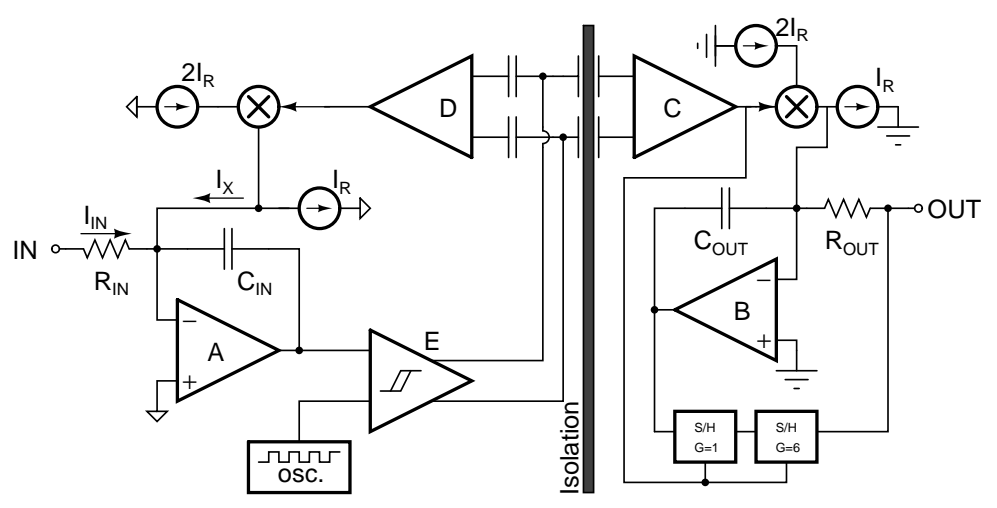

Figure 1: Internal structure of a typical ISO12X according to the manufacturer [6].

as the

\section{Internal Structure of Amplifiers}

This technology was developed by Texas Instruments to build some of its interface devices, either digital or analog. Analog isolation amplifiers make up the ISO12X family and the data shown in this paper were focused on the ISO122 \& ISO124 devices, the datasheets of which can be found on the manufacturer's website [6]. These two devices are quite similar given that the internal block shown in Figure 1 is implemented in both of them. Actually, the only difference between them is that the elementary devices inside the amplifiers such as resistors, capacitors, etc. are more accurately built in the case of the ISO124.

According to the manufacturer, the principle of working is the following: The amplifier $A$ creates a virtual ground at the right side of $R_{I N}$ in such a way that a current $I_{I N}=V_{I N} / R_{I N}$ flows into the isolation amplifier. This signal is added to a current $I_{X}$, the value of which is $\pm I_{R}$ depending on the state of the comparator $D$ that control two current sources, $2 \cdot I_{R} \& I_{R} . I_{X}+I_{I N}$ 


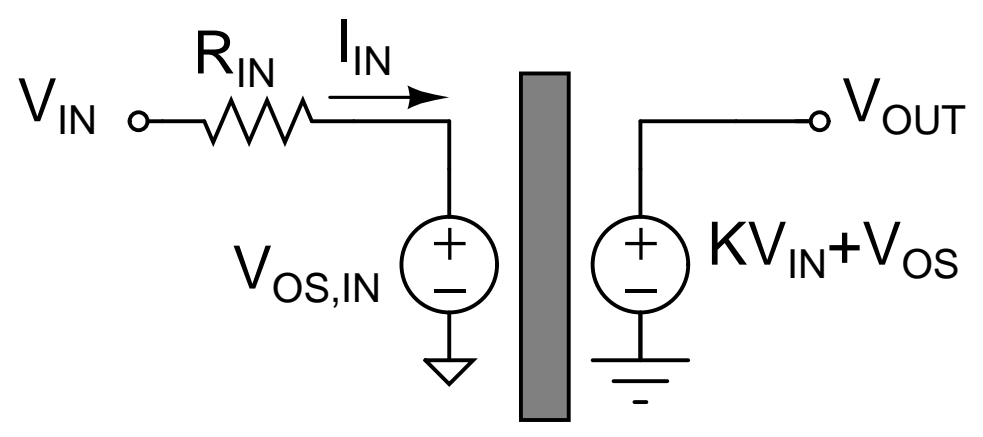

Figure 2: Simplified equivalent macro model of ISO12X, useful for hand analysis.

is used to charge and discharge a capacitor, $C_{I N}$, connected to the output of the amplifier $A$. This node is also connected to a comparator $(E)$ that evaluates the difference between the output of $A$ and a $500-\mathrm{kHz}$ wave generator. Thus, a square signal with a duty cycle depending on the size of $I_{I N}$ and, evidently, on $V_{I N}$ is obtained at the output of $E$.

This signal as well as its complementary is transmitted through the isolation barrier by means of a couple of capacitors so they reach the inputs of another comparator $(C)$, which acts as a buffer to recover the signal. The width-modulated square signal is decoded using several devices in such a way that the initial voltage value is regenerated at the output node of the isolation amplifier.

Unfortunately, it is impossible to reach the internal devices without destroying the isolation amplifier. Thus, a simple macro model (Figure 2) containing as much information as possible was developed to evaluate the degradation of the device and to allow a later use in simulations or hand calculations. In this structure, $R_{I N}$ is the input resistance shown in Figure 1. $V_{O S, I N}$ is the input offset voltage of the $A$ operational amplifier. Ideally, the voltage value at the inverting input of this operational amplifier should be $0 \mathrm{~V}$ but, due to the input offset voltage, the voltage value at this node is not 0 but $V_{O S, I N}[7]$ and can be measured as it will be later shown. The output stage is modeled by means of a voltage-controlled voltage source the gain of which is ideally $K=1$. An additional output offset voltage, $V_{O S}$, is included to take into account non-idealities and other defects of the output stage.

It must be highlighted that the "input offset voltage" given by the manufacturer in the datasheets is just the "output offset voltage" defined in this paper. Actually, the offset voltage in the input operational amplifier only affects the function associating $V_{I N}$ with $I_{I N}$. In other words, the input characteristic. In fact, even though large values of $V_{O S, I N}$ were measured, the output voltage with zero input was very close to $0 \mathrm{~V}$. The input offset voltage could affect the size of the input current 
in such a way that the modulated-width square signal is distorted. However, the decoding of the transmitted information is made using a similar operational amplifier at the output stage. Given that this amplifier has been built in the same wafer as the first one, both devices would be carefully matched so the error introduced by the first amplifier is removed by the second one. Thus, even if the offset voltage of the input operational amplifier is often beyond $100 \mathrm{mV}$, the offset voltage of the complete isolation amplifier never exceeds the typical values provided by the manufacturer $(50 \mathrm{mV})$.

Finally, ideal isolation amplifiers have a transmission coefficient, $K$, equal to 1 . However, in actual devices this value is never accomplished being usually above or beneath this value. An additional parameter, called "typical output error", the meaning of which will be explained later, was also measured along with the transmission coefficient, $K$.

All the parameters depicted in the previous paragraphs were measured on-line but, once the devices could be safely handled, more parameters were measured. Some of these parameters were:

- Power supply rejection ratio $(P S R R)$.

- Insulation between the stages (IMRR, insulating impedance and electric breakdown field).

- Quiescent current, parameter related to the power consumption.

- Frequency behavior

- Output noise

\section{Set-up for the on-line tests}

\subsection{Description of the irradiation facility}

Both kinds of isolation amplifiers were tested at the neutron facility of the Portuguese Research Reactor [8] using three samples of each model. These samples, which belonged to the same batch, were mounted on different printed circuit boards and distributed along a cylindrical cavity with the goal of irradiating each sample with a different total radiation dose. The irradiation took about $20 \mathrm{~h}$ split in three rounds followed by technical reactor shutdown periods. Thus, the samples received the total radiation dose shown in Table 1 . The neutron fluence was obtained with ${ }^{58} \mathrm{Ni}$ foil detectors and multiplied by a factor of 1.27 to express the neutron fluence in standard 1-MeV $\mathrm{n} / \mathrm{cm}^{2}$ units $[5,8]$. The total ionising dose was measured by an ionisation chamber. From now on, 
Table 1: Total radiation dose and dose rate received by the samples.

\begin{tabular}{|c|c|c|c|c|}
\hline Sample & Neutron Fluence & TID & Dose Rate & TID/N.F. \\
\hline $\mathrm{A}$ & 2.20 & 236 & 11.8 & 107.3 \\
\hline $\mathrm{B}$ & 0.95 & 148 & 7.4 & 155.8 \\
\hline \multirow[t]{2}{*}{$\mathrm{C}$} & 0.34 & 104 & 5.2 & 305.9 \\
\hline & $\cdot 10^{13} 1-\mathrm{MeV} \mathrm{n} / \mathrm{cm}^{2}$ & $\mathrm{~Gy}(\mathrm{Si})$ & $\mathrm{Gy}(\mathrm{Si}) / \mathrm{h}$ & $\cdot \mathrm{Gy} / 10^{13} 1-\mathrm{MeV} \mathrm{n} / \mathrm{cm}^{2}$ \\
\hline
\end{tabular}

the total radiation dose will be expressed in units of $1-\mathrm{MeV} \mathrm{n} / \mathrm{cm}^{2}$, the TID value being calculated using the ratios of TID vs. neutron fluence found on Table 1.

The temperature was measured with PT-100 resistive temperature detectors distributed along the facility cavity, which has an injecting-air cooling system so the temperature kept stable around $26-27^{\circ} \mathrm{C}$ during the whole radiation.

\subsection{Acquisition system set-up}

All the printed circuit boards had separated ground for the input \& output stage, and a couple of $\pm 15 \mathrm{~V}$ power supplies to bias the devices. These power supplies were not switched off until the end of the test. During the irradiation, the devices were characterised every ten minutes by an acquisition system consisting in a personal computer, an accurate digitally controlled voltage source, two precision multimeters, and a matrix switching system, all of them controlled by a general purpose interface bus (GPIB). The distance between the samples at the reactor cavity and the instrumentation system was on the order of 3-4 $\mathrm{m}$ so low-resistance shielded pipes were used to connect both parts. It is necessary to say that all the voltages were measured on the boards. This fact is especially important in the case of the input voltage, which was not measured at the input voltage source but directly on the board. Also, the isolation amplifiers were disconnected from the input source and voltmeters using mechanical relays and connected again only during the interval needed to characterise the devices.

This system performed a DC sweep at the input voltage from $-1 \mathrm{~V}$ to $+1 \mathrm{~V}$ with a step of 0.2 $\mathrm{V}$ to obtain the transmission coefficient, $K$, and the output offset voltage, $V_{O S}$, with a linear fit after the data coming from the multimeters.

These linear fits also allowed the calculation of the Typical Output Error $\left(\Delta V_{O U T}\right)$, defined as follows. Supposing that there are $N$ pairs of input and output values $\left(V_{I N, k}, V_{O U T, k}\right)$ that were 


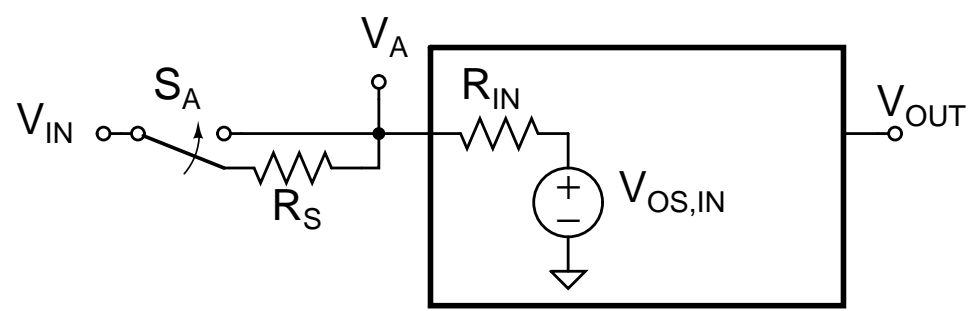

Figure 3: Test set-up to measure $R_{I N} \& V_{O S, I N}$. Using $R_{S}$ makes $V_{A}$ different from $V_{I N}$ and the values of $R_{I N} \&$ $V_{O S, I N}$ are easily extracted.

linearly fitted to obtain the values of $K$ and $V_{O S}, \Delta V_{O U T}$ is:

$$
\Delta V_{O U T}^{2}=\frac{1}{N-2} \sum_{k=1}^{N}\left[V_{O U T, k}-\left(V_{O S}+K \cdot V_{I N, k}\right)\right]^{2}
$$

In order to measure the input offset voltage, $V_{O S, I N}$, and the input resistance, $R_{I N}$, the procedure was as follows: A mechanical relay connects the input source to the input of the isolation amplifier with $R_{S}$, a $10-\mathrm{k} \Omega$ precision resistor (Figure 3 ). A voltage on the order of $+1 \mathrm{~V}$ is set at $V_{I N}$ and a pair of voltages, $V_{I N, 1} \& V_{A, 1}$ are measured and stored. Immediately, the voltage source changes to $-1 \mathrm{~V}$ to measure a new couple of values, $V_{I N, 2} \& V_{A, 2}$. Using Kirchoff's current law it is easy to demonstrate that the values of the unknown parameters are:

$$
\begin{gathered}
V_{O S}=\frac{V_{A, 1}-\alpha \cdot V_{A, 2}}{1-\alpha} \\
\frac{R_{I N}}{R_{S}}=\frac{\alpha}{1+\alpha} \cdot \frac{V_{A, 1}+V_{A, 2}}{V_{I N, 1}-V_{I N, 2}}
\end{gathered}
$$

where

$$
\alpha=\frac{V_{I N, 1}-V_{A, 1}}{V_{I N, 2}-V_{A, 2}}
$$

Initial values of the input resistance are shown in Table 2.

\section{Experimental results and discussion}

\subsection{Transmission coefficient, $K$}

In an ideal isolation amplifier, the transmission coefficient is 1 in order to accurately regenerate the input signal at the output stage. However, actual devices do not accomplish this theoretical 
Table 2: Initial values of the resistance, $R_{I N}$.

\begin{tabular}{ccc} 
Sample & ISO122 & ISO124 \\
\hline A & $196.0 \pm 0.1$ & $199.7 \pm 0.1$ \\
B & $169.9 \pm 0.1$ & $198.3 \pm 0.1$ \\
C & $178.0 \pm 0.1$ & $199.2 \pm 0.1$ \\
\hline & $\mathrm{k} \Omega$ & $\mathrm{k} \Omega$ \\
\hline
\end{tabular}

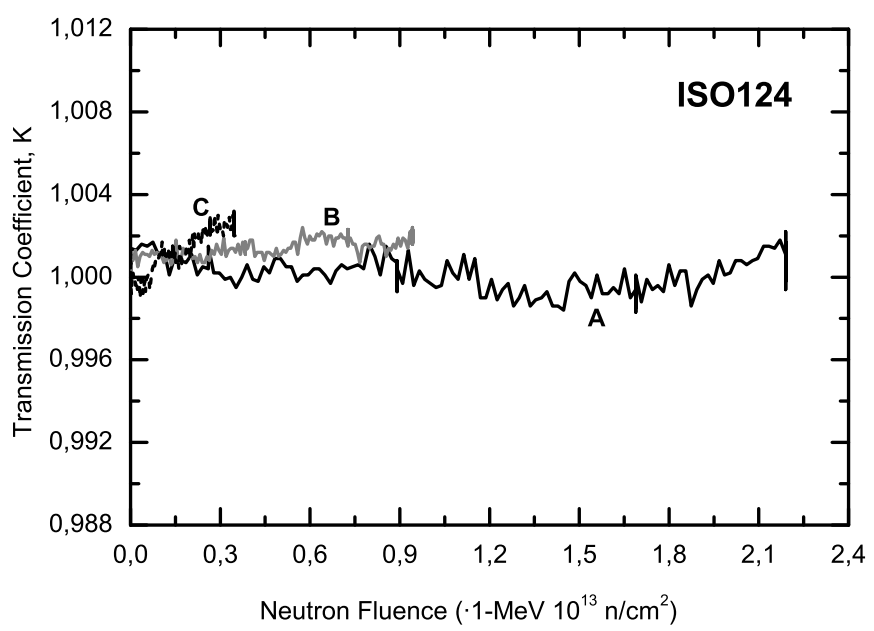

Figure 4: Transmission coefficient of the ISO124.

requirement. In fact, the pristine samples of the ISO122 showed a scattering of this parameter between 1.000 \& 1.004 (0.4\%), this error being smaller in the ISO124 where the transmission coefficient values were between $1.000 \& 1.001$ (0.1\%). Let us remember that the ISO124 is similar to the ISO122 with more accurately trimmed internal components.

This can be the reason of the different behaviour of the transmission coefficient in both devices. Figure 4 shows the evolution of $K$ at the ISO124. The value of this parameter keeps quite stable even at the most irradiated sample and only deviations up to 1.003 were registered in some of the devices. On the contrary, the evolution of $K$ in the ISO122 (Figure 5) is much more problematic given that, a priori, it is impossible to know if this parameter will increase or decrease and that, at any rate, the shift in this parameter makes the value of $K$ be placed between $0.990 \& 1.009$. In other words, the possible error goes beyond $1 \%$.

In the authors' opinion, this different behaviour is a consequence of the worse trimming of 


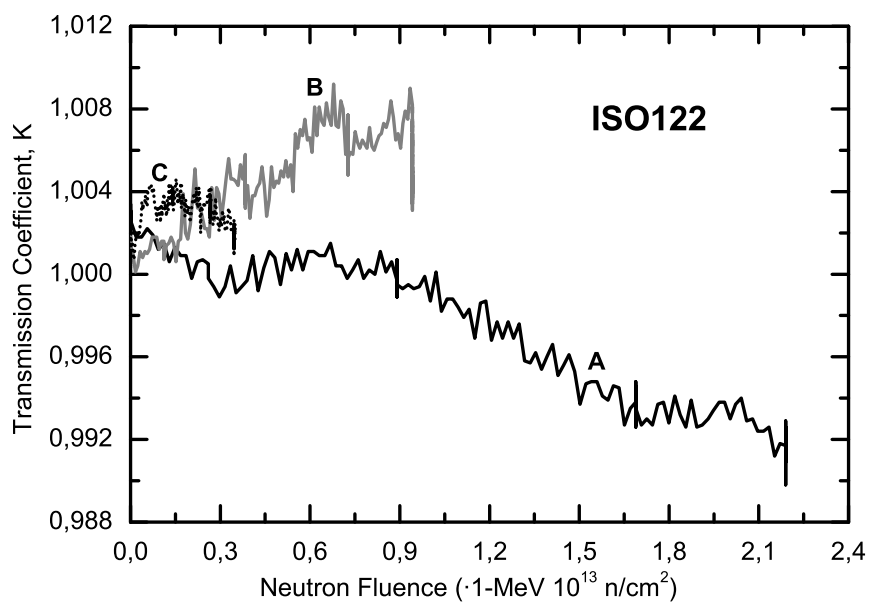

Figure 5: Transmission coefficient of the ISO122.

the internal devices of the ISO122. Probably, the radiation damage accentuates the mismatch between supposed similar devices making the transmission coefficient move away from the ideal value. Given that the internal devices of the ISO124 are better trimmed, the deviation is smaller.

\subsection{Offset Voltage, $V_{O S}$}

Pristine samples of both devices have a typical output offset voltage between $\pm 20 \mathrm{mV}$. Unfortunately, these limits are quickly exceeded in most of the samples. Figures $6 \& 7$ show the exact evolution of this parameter. Some important conclusions can be drawn from these figures. First of all, it is impossible to forecast the exact evolution of the offset voltage since it grows in some devices, decreases in other of them and keeps quite constant in one of the ISO122. In any case, it seems evident that the shift is larger in the ISO122 samples than in the ISO124: The ISO122 offset voltage keeps between -40 \& $140 \mathrm{mV}$ whereas in the ISO124 the limits are wider $(-130$ \& $220 \mathrm{mV})$.

The origin of this difference of behaviour would come from the same fact as the transmission gain. Offset voltages are caused by mismatches among the internal components of a specific device. Radiation damage makes these differences more significant changing the value of the offset voltage. These mismatches are unknown so, provided the great number of parameters involved in the value of the offset voltage, the final evolution is impossible to predict. This evolution of the offset voltage is very similar to that observed in irradiated operational amplifiers, especially those with JFET input stage where the matching is worse than those completely built with bipolar junction 


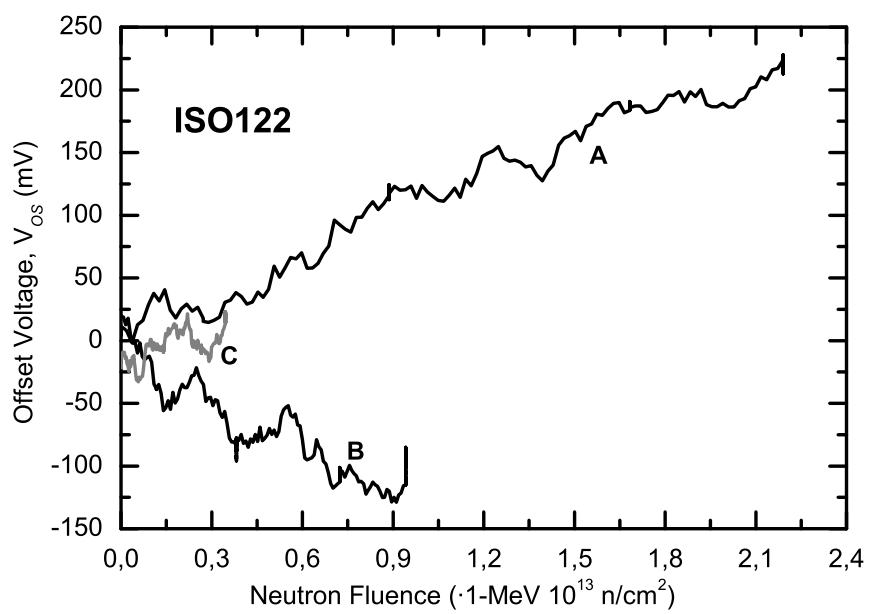

Figure 6: Offset voltage of the ISO122.

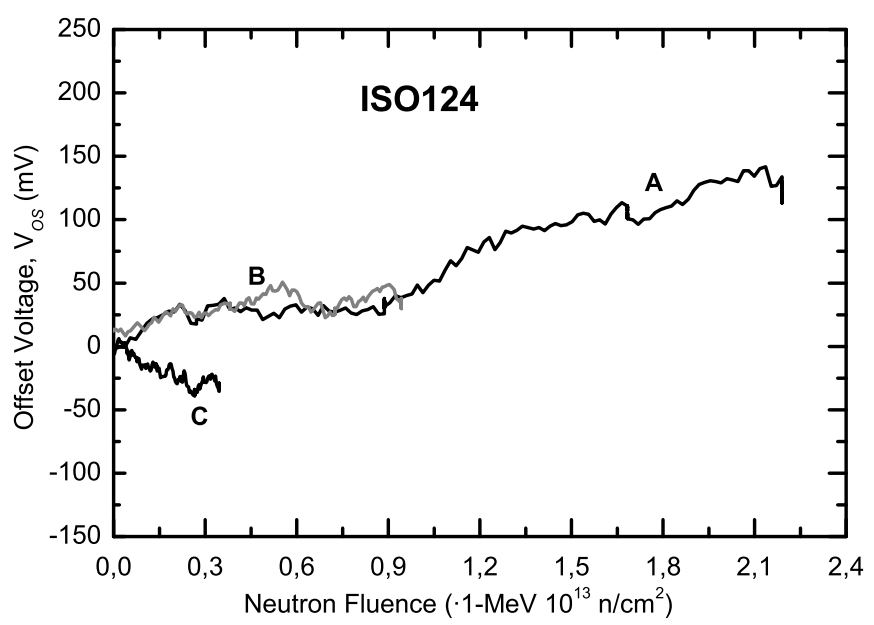

Figure 7: Offset voltage of the ISO124. The Y-axis scale is similar to that of the ISO122 (Figure 6) to make the comparison between both of them easier. 


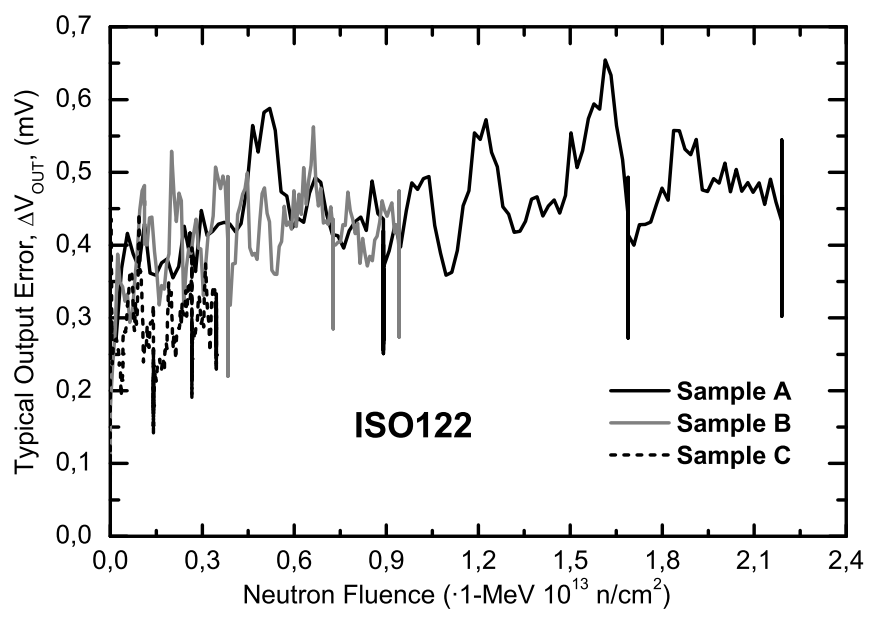

Figure 8: Typical output error of the ISO122, $\Delta V_{O U T}$.

transistors $[9,10,11,12,13,14]$.

From the system designer's point of view, the large values of the offset voltage are a serious concern. Fortunately, there are some state-of-the-art techniques that minimise this drawback, such as that depicted in [15].

\subsection{Typical Output Error, $\Delta V_{\text {OUT }}$}

In ideal isolation amplifiers, the value of this parameter is $0 \mathrm{~V}$, situation that is never achieved in actual devices due to the output noise and the nonlinearity of the device.

Before the tests, samples of the ISO122 showed a value of $\Delta V_{\text {OUT }}$ about $0.25 \mathrm{mV}$ while the other device showed a lower value, $0.1 \mathrm{mV}$. The evolution of this parameter is shown in Figures 8 \& 9 from which we can see that the value of $\Delta V_{O U T}$ increases as the irradiation is carried out. In the case of the ISO122, the highest value is on the order of $0.65 \mathrm{mV}$, being lower in the case of the ISO124, where the value of $\Delta V_{\text {OUT }}$ never went beyond $0.45 \mathrm{mV}$.

The reason of this behaviour is not completely understood. It is well-known that all the irradiated electronic devices show a higher noise level due to the creation of defects inside the silicon lattice. However, the complexity of the device does not allow accepting that this is the only cause. E. g., ionising radiation can create finite impedance paths below the epitaxial oxide allowing the interferences of any of the $500-\mathrm{kHz}$ signals at the output node. 


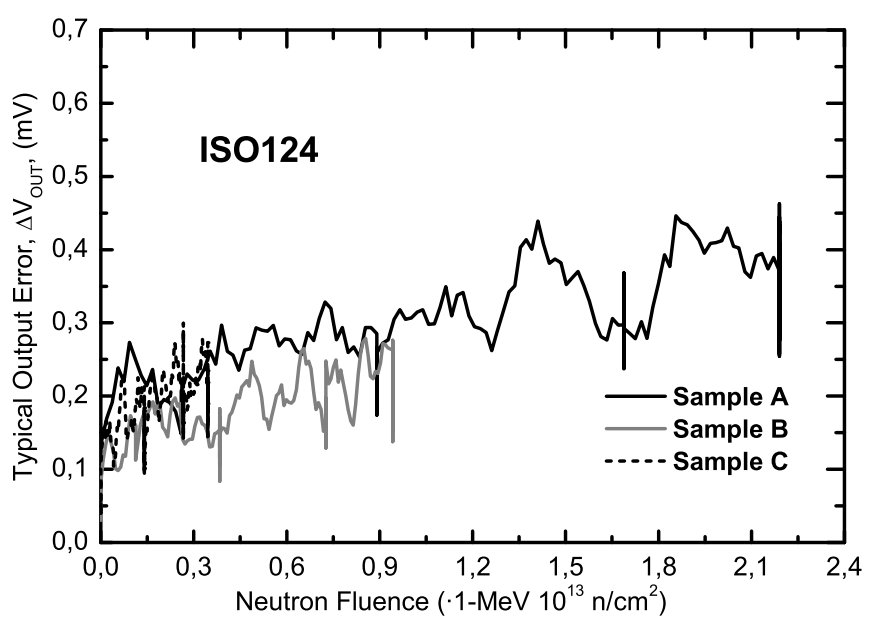

Figure 9: Typical output error of the ISO124, $\Delta V_{O U T}$.

4.4. Input resistance, $R_{I N}$

According to the manufacturer, the value of this parameter is $200 \mathrm{k} \Omega$. However, actual devices do not accomplish this requirement (Table 2). In fact, the value of $R_{I N}$ in the ISO122 varies from 170 to $200 \mathrm{k} \Omega$ although, in the case of the ISO124, the variation range is much smaller (198-200 $\mathrm{k} \Omega$ ). This fact is clearly related to the more careful process used by the manufacturer to build this model.

Concerning the effects of the radiation, no change was observed during the tests since their values kept constant until the end. Figure 10 shows the behaviour of the ISO124 input resistances as the irradiation was performed. The fact that the input resistance are implemented in metallic thin-film resistor technology explains the great tolerance of this part of the isolation amplifiers since it is commonly accepted that metals are insensitive to either displacement or ionisation damage [16].

\subsection{Offset voltage of the input operational amplifier, $V_{O S, I N}$}

The values of these parameters were initially distributed between $\pm 150 \mathrm{mV}$ in all the tested devices. Unlike the offset voltage, the change was steady and monotonic without a constant shift rate that could strongly vary from one sample to another (Figure 11). For instance, the most irradiated sample of the ISO122 showed a shift rate of $1.02 \mathrm{mV} / 10^{13} \mathrm{n} / \mathrm{cm}^{2}$ while, in the second sample of the same device, the ratio was $-9.44 \mathrm{mV} / 10^{13} \mathrm{n} / \mathrm{cm}^{2}$, more than nine times larger. 


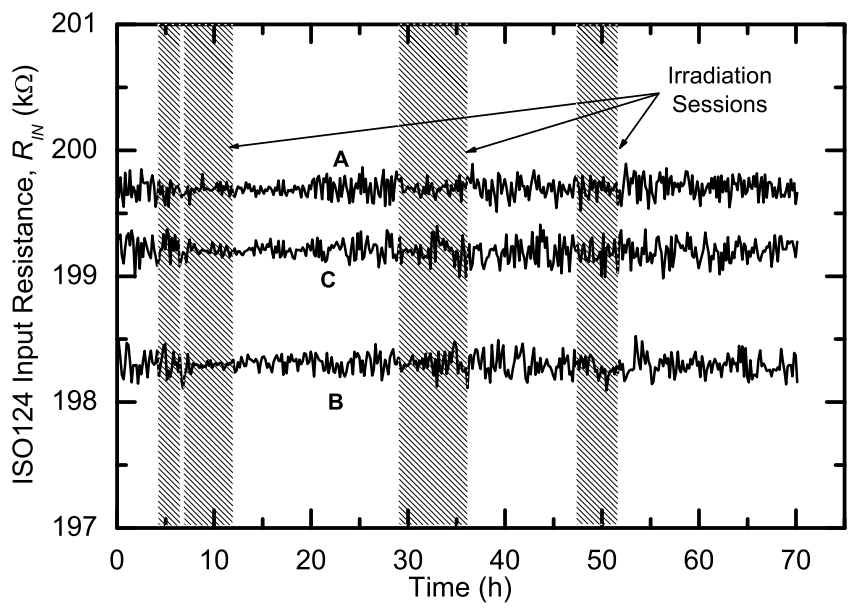

Figure 10: Input resistances of the ISO124 during the irradiation. Because of unknown reasons, the noise level is greater during some periods.

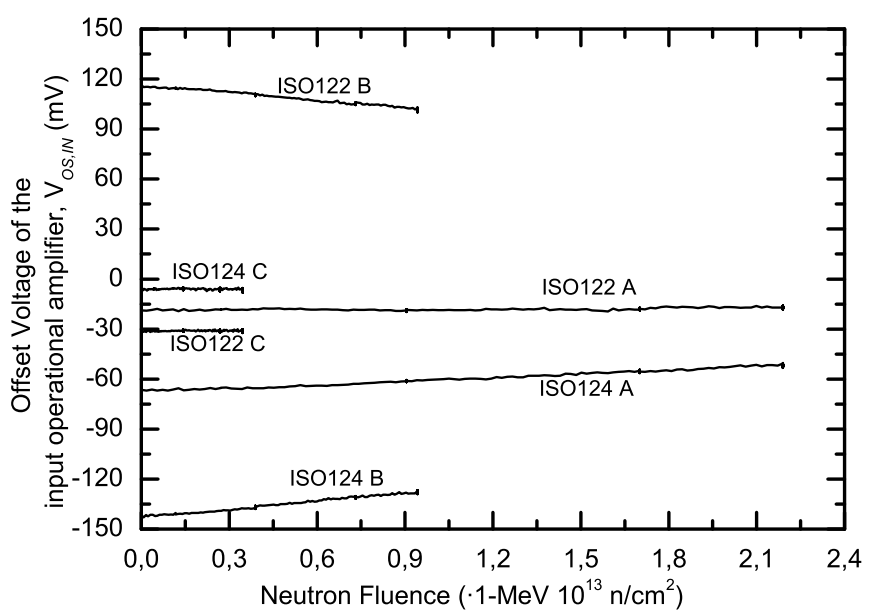

Figure 11: Offset voltage of the input operational amplifier during the irradiation. 


\subsection{Off-line parameters}

Other parameters could not be measured during the test. They were taken about one month later once that the radioactive isotopes generated during the irradiation had vanished and a safe handling could be done.

\subsubsection{Power supply rejection ratio (PSRR)}

This parameter measures the dependence of the offset voltages with the power supply values. In the case of $V_{O S, I N}$, no significant change was found. In the case of the output offset voltage, the high noise level typical of these devices masked the little variations of this parameter needed to estimate the experimental PSRR value.

\subsubsection{Isolation barrier}

The main characteristic of these devices is the ability to insulate input and output stages. Therefore, it is essential to verify the integrity of the isolation barrier.

The isolation mode rejection ratio $(I M R R)$ is a parameter that evaluates the influence of the common-mode voltage, $V_{I S O}$, on the output and is defined as:

$$
I M R R=\frac{\partial V_{O U T}}{\partial V_{I S O}}
$$

In order to measure it, a common-mode voltage between $\pm 1000 \mathrm{~V}$ was applied between the stages with the purpose of measuring the slight output voltage variations. However, and as it occurred with the PSRR, the noise was so significant that we could only estimate that the IMRR was always larger than $120 \mathrm{~dB}$ in the pristine samples of both kinds of amplifiers. After the test, even the most irradiated samples did not show a lower value.

To account for these results, we proceeded to calculate the value of the impedance between both stages. This impedance is modeled just as a resistor and a capacitor in parallel. The resistor was measured with a Kyoritsu high voltage insulation tester with the final conclusion that its value was, at least, higher than $100 \mathrm{~T} \Omega$ in all the samples (This value is the upper measure limit of the instrument).

The capacitor between the isolated grounds was measured by means of a Hewlett Packard 4192 impedance analyser in the $10-100 \mathrm{kHz}$ frequency range, the results being shown in Table 3 . The first fact to bear in mind is that the actual value of the capacitors is higher than that specified by the manufacturer $(2 \mathrm{pF})$. However, in any case they are very close so the discrepancy can be 
Table 3: Total radiation dose and dose rate received by the samples.

\begin{tabular}{cccc} 
Sample & Neutron Fluence & ISO122 & ISO124 \\
\hline- & 0.00 & $3.3 \pm 0.2$ & $3.4 \pm 0.2$ \\
$\mathrm{~A}$ & 2.20 & $3.2 \pm 0.2$ & $3.2 \pm 0.2$ \\
$\mathrm{~B}$ & 0.95 & $3.5 \pm 0.2$ & $3.3 \pm 0.2$ \\
$\mathrm{C}$ & 0.34 & $3.2 \pm 0.2$ & $3.2 \pm 0.2$ \\
\hline & $\cdot 10^{13} 1-\mathrm{MeVn} / \mathrm{cm}^{2}$ & $\mathrm{pF}$ & $\mathrm{pF}$ \\
\hline
\end{tabular}

attributed either to little errors during the manufacture or to the appearance of parasitic capacitors not included in the simplified ISO12X schematic. The second conclusion from Table 3 is that there is no evidence of modification of the capacitance since differences among the values in this table are always within the experimental error.

Finally, the tolerance of the irradiated isolation barrier to very strong electric fields was tested applying a high voltage between the ground pins of each stage. The manufacturer guarantees the tolerance of these devices to common-mode voltages below $1500 \mathrm{~V}$, fact that was confirmed by our measures. Indeed, we applied a common-mode voltage between $\pm 5000 \mathrm{~V}$ for a few seconds without destroying even the most irradiated samples.

\subsubsection{Quiescent currents}

Before the irradiation, the typical quiescent current was on the order of 4.8-4.9 mA for each stage using $\pm 15 \mathrm{~V}$ power supplies. Later, we observed a little decrease in this parameter. Thus, the most irradiated sample of the ISO124 only required $4.15 \mathrm{~mA}$ to work.

\subsubsection{Frequency behavior}

Figure 12 shows the dependence on the frequency value of the transmission coefficient obtained from the second sample of the ISO124. The gain was measured applying an input sinusoidal signal of an r.m.s. value of $100 \mathrm{mV}$ and measuring the r.m.s output voltage. This sample was selected to be represented since it was that one where the degradation was greater. Figure 13 shows the evolution of the characteristic frequencies of the ISO124 samples. Similar results were found on the ISO122.

Radiation damage always causes degradation in the frequency response of operational amplifiers and derived devices [17]. Therefore, it is possible that the degradation observed in the devices is 


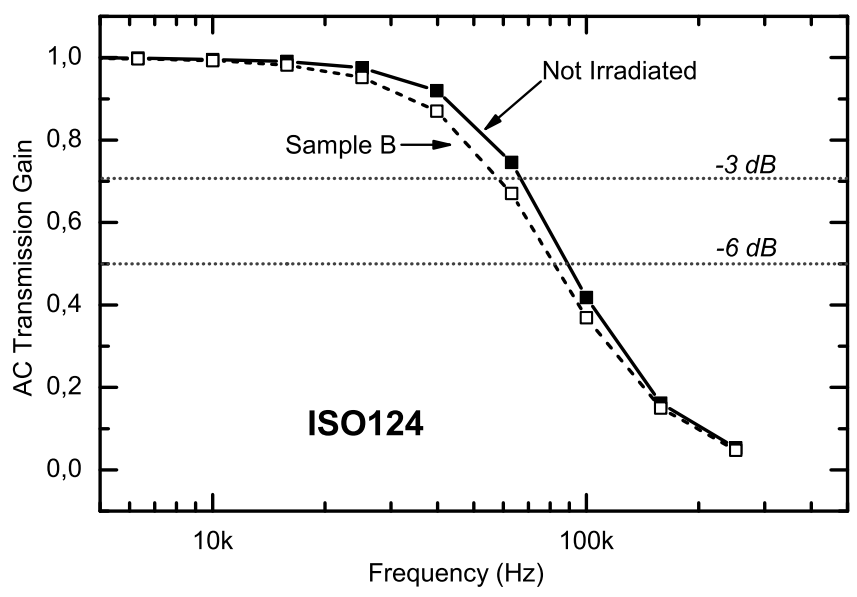

Figure 12: Bode diagram with linear Y-axis of the second sample of the ISO124. Pre and post irradiation lines can be found in the plot. The sample received $0.95 \cdot 10^{13} 1-\mathrm{MeV} \mathrm{n} / \mathrm{cm}^{2}$.

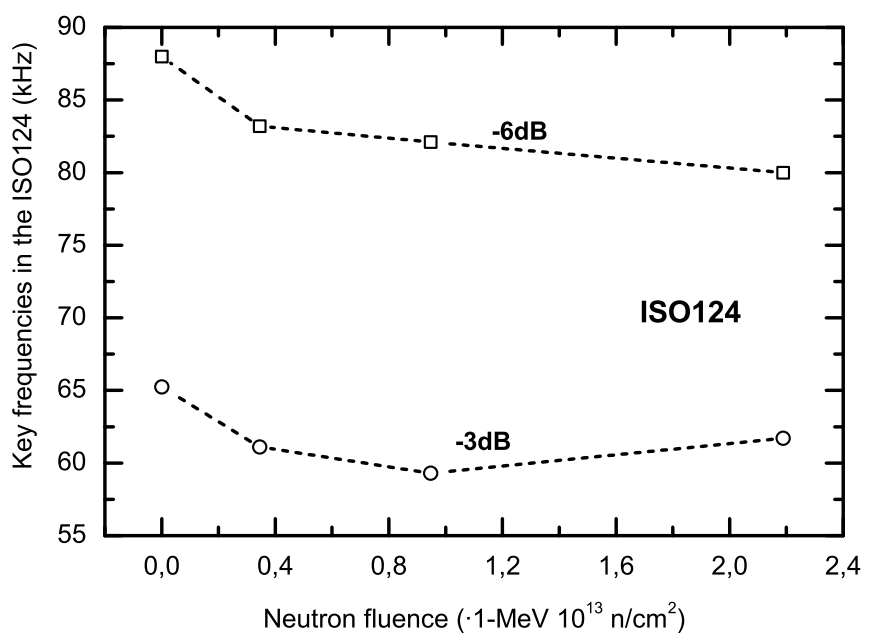

Figure 13: Frequency values where decreases of $3 \& 6 \mathrm{~dB}$ were observed in the irradiated ISO124. 


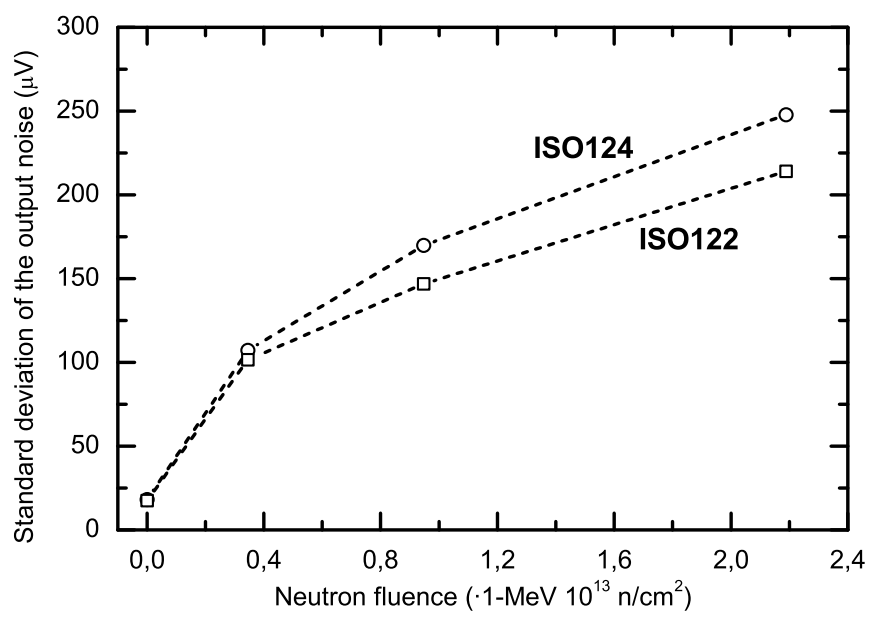

Figure 14: Standard deviation of the output voltage, $\sigma$, related to the intrinsic output noise. Supposing that the theoretical value of the output voltage is $V_{M E A N}$, most of the times the actual output voltage would be a random value between $V_{M E A N} \pm 2 \cdot \sigma$.

a manifestation of the slower response of the internal devices such as operational amplifiers or comparators. Nevertheless, this could not be the only reason: Isolation amplifiers give up being linear devices at high frequency. Provided that the modulator-demodulator works at $500 \mathrm{kHz}$, the Nyquist frequency is $500 / 2=250 \mathrm{kHz}$. This is the threshold that limits the correct work of sampling amplifiers, usually being even lower than the theoretical value [18]. Therefore, the degradation of the frequency response could also be related to a decrease on the oscillator frequency in such a way that the Nyquist frequency effects appear at lower frequency values.

\subsubsection{Output noise}

This parameter is closely related to the typical output error and was measured following this method. The device was biased with noise-free power supplies and the input connected to ground. After waiting for a few minutes in order to stabilise the output, a high-accuracy multimeter measured the output voltage 1000 times in less than one minute. The output voltage usually shows a random drift that was calculated using a $9^{\text {th }}$-order Savitzky-Golay filter and later removed from the output signal. Thus, the output voltage was centered on about $0 \mathrm{mV}$ and the values distributed following a typical Gauss bell. In such a situation, the output noise is easily described by means of the standard deviation.

Figure 14 shows the evolution of this parameter in both kinds of devices. According to these 
results, the output noise voltage soars from $20 \mu \mathrm{V}$ up to $200-250 \mu \mathrm{V}$ in the most irradiated samples. These results are on the order of the typical output error but always lower. There are several factors that explain this behavior. First of all, output noise were measured one month later so the devices partially recovered by natural annealing. Second, during the on-line tests the devices were several meters far from the instrumentation system so the measurements were not as accurate as those performed at the laboratory. Finally, the intrinsic non-linearities of the isolation amplifiers do not affect the output noise since it was measured with an only input value $(0 \mathrm{~V})$ whilst the typical output error was extracted from a DC sweep.

\section{Conclusion}

Capacitor-based isolation amplifiers undergo degradation if they are exposed to radiation. However, they can keep operative even when the total radiation dose reaches a value of $2.4 \cdot 10^{13} 1-\mathrm{MeV}$ $\mathrm{n} / \mathrm{cm}^{2} \& 235 \mathrm{~Gy}(\mathrm{Si})$. In this situation, the most affected parameters are the offset voltages, the transmission gain and the typical output error. Therefore, in case of wishing to use capacitor-based isolation amplifiers in instrumentation systems under radiation, some rules must be followed in order to guarantee the accuracy of the whole system. Techniques to remove the offset voltage must be integrated in the design along with low-pass filters to attenuate the output noise. Besides, the system must be able to correct the isolation amplifier gain drift. Finally, precision versions seem to be more radiation-tolerant than the devices with lower quality.

\section{Acknowledgements}

This work was supported by the cooperation agreement K476/LHC between CERN \& UCM, by the Spanish Research Agency CICYT (FPA2002-00912), by the Spanish Agency for the International Cooperation (AECI) and by the Miguel Casado San José Foundation.

\section{References}

[1] M. Rowe, Isolation boosts safety and integrity, Test \& Measurement World. URL http://www.reed-electronics.com/tmworld/article/CA235286

[2] A. J. Peyton, V. Walsh, Analog Electronic with Op Amps: A Source Book of Practical Circuits, Cambridge University Press, Cambridge (UK), 1993, iSBN: 0-521-3305-X. 
[3] G. Messenger, M. Ash, The Effects of Radiation on Electronic Systems, 2nd Edition, Van Nostrand Reinhold, New York City (USA), 1992, iSBN: 0-442-23952-1.

[4] V. Remondino, Radiation damage in amplifiers used for quench detection in a superconducting accelerator, in: Proceedings of the $3^{\text {rd }}$ European Conference on Radiation and its Effects on Components and Systems (RADECS95), RADECS Association, French Space Agency (CNES), Arcachon (France), 1995, pp. 89-93, iSBN: 0-7803-3093-5. doi:10.1109/RADECS.1995.509757.

[5] Y. Zong, F. J. Franco, J. A. de Agapito, A. C. Fernandes, J. G. Marques, Radiation tolerant isolation amplifiers for temperature measurement, Nuclear Instruments and Methods in Physics Research Section A: Accelerators, Spectrometers, Detectors and Associated Equipment 568 (2) (2006) 869-876. doi:10.1016/j.nima.2006.09.007.

[6] Texas instruments website, On-line at http://www.ti.com.

[7] B. Carter, R. Mancini, Op Amps for Everyone, 3rd Edition, Texas Instruments, USA, 2009, Ch. 13: Understanding Op Amp Parameters, pp. 220-221, iSBN: 978-1-85617-505-0.

[8] J. G. Marques, A. C. Fernandes, I. G. Gonçalves, A. J. G. Ramalho, Test facility at the portuguese research reactor for irradiations with fast neutrons, in: E. Ragel, R. Tamayo, C. Sánchez (Eds.), Proceedings of the $5^{t h}$ Workshop on Radiation Effects on Components and Systems (RADECS04), RADECS Association, Instituto Nacional de Tecnica Aeroespacial (INTA), Madrid (Spain), 2004, pp. 335-338, iSBN 84-930056-1-4.

[9] C. Lee, B. Rax, A. Johnston, Hardness assurance and testing techniques for high resolution (12 to 16-bit) analog-to-digital converters, IEEE Transactions on Nuclear Science 42 (6) (1995) 1681-1688, iSSN: 0018-9499. doi:10.1109/23.488766.

[10] L. Bonora, J. David, An attempt to define conservative conditions for total dose evaluation of bipolar ics, IEEE Transactions on Nuclear Science 44 (6) (1997) 1974-1980, iSSN: 0018-9499. doi:10.1109/23.658972.

[11] C. Lee, A. H. Johnston, Comparison of total dose effects on micropower op-amps: bipolar and cmos, in: Proceedings of the IEEE Radiation Effects Data Workshop, IEEE Nuclear and 
Plasma Sciences Society, The Institute of Electrical and Electronics Engineers (IEEE), Inc., Newport Beach, CA, USA, 1998, pp. 132-136. doi:10.1109/REDW.1998.731492.

[12] R. Pease, M. Gehlhausen, J. Krieg, J. Titus, T. Turflinger, D. Emily, L. Cohn, Evaluation of proposed hardness assurance method for bipolar linear circuits with enhanced low dose rate sensitivity (eldrs), IEEE Transactions on Nuclear Science 45 (6) (1998) 2665-2672, iSSN: 0018-9499. doi:10.1109/23.736512.

[13] R. Pease, J. Krieg, M. Gehlhausen, D. Platteter, J. Black, Total dose induced increase in input offset voltage in jfet input operational amplifiers, in: Proceedings of the $5^{\text {th }}$ European Conference on Radiation and its Effects on Components and Systems (RADECS99), RADECS Association, French Atomic Energy Commission (CEA), Fontevraud (France), 1999, pp. 569572, iSBN: 0-7803-5726-4. doi:10.1109/RADECS.1999.858649.

[14] D. M. Hiemstra, High total dose performance of various commercial off the shelf operational amplifiers during irradiation, in: Proceedings of the IEEE Radiation Effects Data Workshop, IEEE Nuclear and Plasma Sciences Society, The Institute of Electrical and Electronics Engineers (IEEE), Inc., Reno, Nevada (USA), 1999, pp. 32-38, iSBN: 0-7803-6474-0. doi:10.1109/REDW.2000.896266.

[15] J. Agapito, J. Casas-Cubillos, F. Franco, B. Palan, M. Rodriguez Ruiz, Rad-tol field electronics for the lhc cryogenic system, in: Proceedings of the $6^{\text {th }}$ European Conference on Radiation and its Effects on Components and Systems (RADECS03), RADECS Association, European Space Agency (ESA), Noordwijk aan Zee (The Netherlands), 2003, pp. 653-657, iSSN: 03796566, ISBN: 92-9092-846-8.

[16] J. Srour, J. McGarrity, Radiation effects on microelectronics in space, Proceedings of the IEEE 76 (10) (1988) 1443-1469, iSSN: 0018-9219. doi:10.1109/5.90114.

[17] F. Faccio, Cots for the lhe radiation environment: The rules of the game, in: Proceedings of the $6^{\text {th }}$ Workshop on Electronics for the LHC Experiments (LEB2000), Krakow (Poland), 2000, pp. 50-63.

[18] P. Horowitz, W. Hill, The Art of Electronics, 2nd Edition, Cambridge University Press, United States of America, 1990, Ch. 13, pp. 900-902, iSBN:0-521-37095-7. 